\title{
Conformity Behavior in Group Playlist Creation
}

Christine Bauer

Bruce Ferwerda

Johannes Kepler University Linz Jönköping University

Institute of Computational

Perception \& LIT AI Lab

Linz, Austria

christine.bauer@jku.at
Department of Computer

Science and Informatics

Jönköping, Sweden

bruce.ferwerda@ju.se

\begin{abstract}
A strong research record has evidenced that individuals tend to conform with a group's majority opinion. In contrast to existing literature that investigates conformity to a majority opinion against an objectively correct answer, the originality of our study lies in that we investigate conformity in a subjective context. The emphasis of our analysis lies on the "switching direction" in favor or against an item. In an online experiment, groups of five had to create a music playlist. A song was added to the playlist with an unanimous positive decision only. After seeing the other group members' ratings, participants had the opportunity to revise their own response. Results suggest different behavior for originally favored compared to disliked songs. For favored songs, one negative judgement by another group member was sufficient to induce participants to downvote the song. For disliked songs, in contrast, a majority of positive judgements was needed to induce participants to switch their vote.
\end{abstract}

\section{Author Keywords}

Conformity behavior; social influence; music playlist creation; group music playlists; group recommendation.

\section{CCS Concepts}

-Human-centered computing $\rightarrow$ User studies; Empirical studies in $\mathrm{HCl}$; $\cdot$ Applied computing $\rightarrow$ Psychology; -Information systems $\rightarrow$ Recommender systems; 


\section{Introduction}

Social influence and conformity have been studied in faceto-face situations for a long time [32]. While social influence has been studied in online settings as well [40, 39], conformity has received far less attention [32]. Most online conformity research focuses on group norms in online communities (e.g., [35, 28]) or expression in online reviews (e.g., [16]). Yet, other forms of online group scenarios deserve attention, too. Algorithmic decision-making for groups, for instance, is an increasingly important topic (e.g., [21, 34])

A special form of algorithmic decision-making for groups are so-called group recommender systems [26] that compute the most relevant item(s) (e.g., movies to be watched, vacation packages) for the whole group. A particular challenge of group recommenders is to consolidate the various, possibly contradicting, preferences of the various group members [26, 13]. While studies investigating conformity typically follow a study design where participants have to decide between correct and wrong answers, group recommenders operate on taste and preferences where none of the decisions is objectively correct or wrong. Yet, conformity in such settings has not been investigated in depth.

We address this research gap and present first results of our study on conformity, which is part of our ongoing research on group recommender systems. Our online experiment where groups had to create a music playlist contributes to the following research question: Whether and how do people conform in a group-decision setting of preferences and taste?

This paper is structured as follows: First, we present the conceptual basis and discuss related work. Then, we detail the study design. After reporting the results, we discuss the findings and implications, and point to future research.

\section{Conceptual Basis and Related Work}

Social influence refers to the change in an individual's thoughts, feelings, attitudes, or behavior resulting from the interaction with another individual or a group [37]. Responses to social influence may take forms of conformity or nonconformity [27]. In this work, we focus on conformity which is a concept from social psychology and was coined by Asch [1, 2, 3]. It refers to the phenomenon that individuals tend to forgo their personal strategy (e.g., opinion, preference) and adopt the conflicting majority variant [36].

\section{Studies on Conformity}

In context of conformity, Deutsch and Gerard [9] distinguish informational and normative influence. Informational influence occurs if an individual adopts the thoughts and attitudes from the social environment as their own [37]. Often, the social environment is used as guidance in uncertain situations [17] in an attempt to be right [38]. Normative influence, in contrast, describes that an individual expresses a particular opinion or behavior in order to fit the given social environment without necessarily holding that opinion [37]. Here, conformity is based on a goal of obtaining social approval [32] and an attempt to fit in with a group [38].

The most influential study of conformity goes back to Asch [1, $2,3]$. In his experiments, a significant proportion of participants (33.3\%) revised their individual judgements to agree with a clearly incorrect, yet unanimous majority. Asch's study design (i.e., a line judgement task) was used by an extensive number of studies (for a meta-analysis see [4]). Crutchfield [8] took a similar paradigm, yet removing the face-to-face situation and varying the tasks to be performed (e.g., including logical tasks and expressions of attitudes). One major finding of conformity research is that individuals tend to change their personal judgements and opinions when challenged by an opposing majority $[1,4]$. 


\section{Sidebar 1: \\ Computation of Bots}

The decisions of the bots were programmed in such a way that for the initial response each bot had a $30 \%$ chance to vote for a song in a similar fashion as the participant and $70 \%$ chance against. For the

final response, bots were programmed with a $50 / 50$ chance of only changing in the sub-scale of their initial response (i.e., yes/maybe yes or no/maybe no). For the bots, no complete switch in the vote happened.

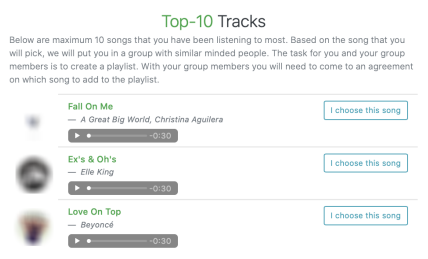

Figure 1: Screenshot with a participant's most played songs for choosing one seed song.

\section{Sidebar 2: \\ Spotify API \\ https://developer.spotify.com/}

\section{Studies on Conformity in Online Settings}

Results from studies on conformity in computer-mediated scenarios vary to a great extent. When following the procedure of Asch's original line judgment task in a computermediated setting, the majority influence disappeared in an early study [33], whereas it was clearly observable in later studies, though demonstrating lower effects when compared to a face-to-face condition [6]

Furthermore, in face-to-face settings, individuals from collectivistic cultures were found to manifest greater levels of conformity than those from individualistic cultures [5]; yet, this effect could not be observed in a computer-mediated setting when using Asch's study design [6]. Still, online studies investigating conformity outside Asch's paradigm found similar cultural effects to the ones observed in faceto-face settings. For instance, when writing online reviews, consumers from collectivistic cultures are less likely to deviate from the average prior rating in their own reviews [16]

Further studies outside Asch's paradigm have investigated various forms of conformity in online settings. Results indicate that depersonalization and anonymity may lead to a more extreme perception of group norms [20] and may encourage to more strongly conform to those [29, 30]. Studies on social media [25, 24] observed that people tend to adopt the majority's opinion on social or political issues. A recent study [38] found that the level of conformity to the majority increased as the difference between the majority size and the minority size increased. A study with mixed groups of human and nonhuman agents [15] found different levels of conformity depending on group composition and task type. Carrying out an emotion judgment task led to higher levels of conformity with the group opinion as the number of humans in the group increased. When performing arithmetic operations, such an effect has not been observed.
Studies on Conformity and Music

Studies on conformity related to music preferences are scarce. Inglefield [18] (cited in [14]) found that differences in perceived peer group membership affected changes in preferences across musical styles. Investigating conformity concerning music preferences, Furman and Duke [14] found that participants unfamiliar with orchestral music were significantly influenced by the others' judgements, whereas no conformity effect was observed for participants familiar with such music. With the same study design but for pop music, in contrast, no such effects have been observed.

In an online music listening setting, a study [12] found that feedback-irrespective of the source-significantly influenced participants' judgements, where feedback from other individuals was more influential than feedback allegedly based on a computational analysis of the music. Another study [10] found that popularity influence (i.e., driven by an item's overall popularity in the whole community) and proximity influence (i.e., driven by an item's popularity in the immediate social network of friends) are substitutes for one another. Yet, when both are available, proximity influence dominates the effect of popularity influence.

Social Influence and Recommender Systems

A system's recommendations may affect users' opinions on the items [7], and also social influence plays a crucial role. For instance, people tend to reverse their ratings when confronted with other people's ratings [41].

As social factors play an important role in group recommenders [31], there is research on algorithmic mechanisms that account for such factors. For instance, [11] identify group leaders and give respective weight to their preferences in a group music recommender. In a movie recommender, [22] anticipate conformity dynamics in their conformity modeling technique. 


\section{Sidebar 3: \\ Configuration of the Popu- larity Parameter \\ Song suggestions were pro- vided based on the selected seed song by using the pop- ularity parameter of 25 or 75. The popularity parameter switched when participants provided the same initial response to a song for five consecutive times to in- crease chances of different initial responses.}

\section{Sidebar 4:}

\section{Liked and Disliked Songs}

The study design required

any participant to encounter both scenarios-initially liked and initially disliked songs.

The "get recommendations" endpoint allowed to retrieve songs aligned (or not) to a particular participant's music preferences. A setting with a randomly selected set of songs for all participants would not have accounted for the participants' specific preferences and would have borne a high probability that many participants would not have encountered a song that they initially liked.

\section{Study Design}

In this paper, we present first results of an online experiment where groups of five had to create a group playlist. A majority size of three is sufficient for the full conformity impact [1]. To have full control on the group decisions, the only real person in the group was the participant. Responses of the four bots were calculated given a certain chance (see Sidebar 1 for details on bots programming).

The study started with an introduction to the purpose of the study: to investigate how groups of people create music playlists. After that, we asked participants to provide us access to their Spotify listening history by using the Spotify API (Sidebar 2). By using the "top" endpoint of the participant's Spotify account, we were able to retrieve their top-10 most listened songs. We asked participants to pick one of the top-10 as a seed song (Figure 1) to find (fictitious) group members with a similar music taste, and to find songs to suggest for the playlist. We used the selected seed song to retrieve song suggestions through the "get recommendations" endpoint. By differing the popularity parameter (Sidebar 3), we were able to suggest songs with different chances to be initially favored or disliked by a particular participant (Sidebar 4).

Upon presenting a suggested song for the playlist, participants were asked whether they were familiar with the artist and the song, and whether they would like to have the respective song as a candidate for the group playlist (Figure 2). The response options were yes, maybe yes, maybe no, and no. Participants were then put on hold for a random 5-10 seconds for all group members to provide their anonymous response. While presenting the anonymous responses of the group members, participants were asked whether they wanted to change their initial response (Figure 3 ) and were informed that in the next step all identities with the corresponding final responses would be revealed. Displaying the anonymous group responses in the first step ensured that the study only factors in the concept of "majority size" and that other confounding variables such as gender of group members (e.g., [38]) are avoided. With revealing the identities after the final response, we minimized the depersonalization and anonymity effects observed in earlier online conformity research (e.g., [29, 30]). After a final decision had been made and the whole group agreed to add the song to the playlist (i.e., unanimous decision), the song was added (Figure 4); otherwise, the experiment continued without adding the respective song. Participants were given another song to rate until a playlist of 10 songs was created through an unanimous decision-making with the group (the study came to an end as well when more than 30 songs were passed without coming to a consensus of 10 songs for the playlist).

\section{Results}

We recruited 96 participants via Amazon Mechanical Turk (MTurk). Participants were selected based on their Human Intelligence Task (HIT) score with at least 1000 HITs completed and a success rate of $95 \%$. After cleaning the data based on responses to attention questions, we ended with 2047 valid responses of 93 participants. Of those responses, 574 responses were initially negative to adding the suggested song to the playlist and 1473 were positive.

To investigate the conformity effect on the initial responses of participants, two repeated measure ANCOVAs (one on the initial positive and one on the initial negative responses) were conducted to analyze how group responses influence individuals' final decision-making.

A first repeated measures ANCOVA was conducted on the initially favored songs for the playlist (i.e., a yes or maybe 


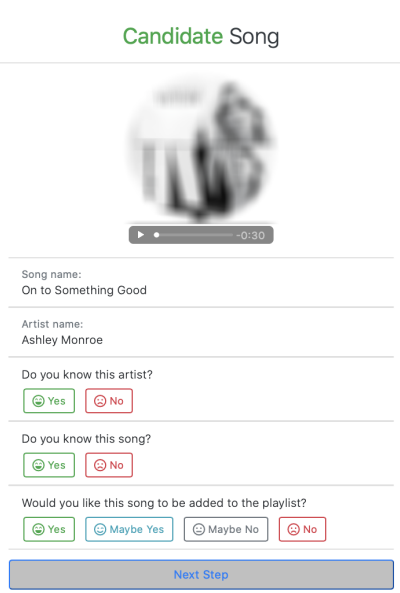

Figure 2: Screenshot with candidate song to be added to the playlist.

Anonymous Group Responses Remember that an unanimous positive fi.e., "yes" or "maybe
yes" decision need to be reached before a song will be added
to the playlish.

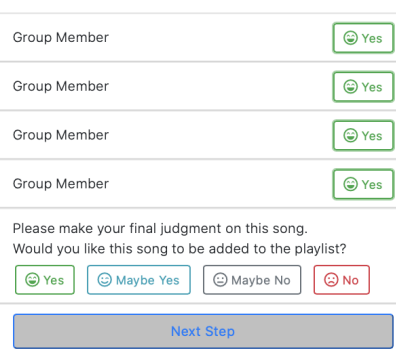

Figure 3: Screenshot showing the group's votes, giving the participant the opportunity to revise their voting. yes response). The Greenhouse-Geisser correction determined that mean responses on a song differed statistically significantly between time points (i.e., before and after presenting the group responses): $F(1, .428)=35.730, p<$ 0.0005 , as well as when considering the group responses through the interaction effect: $F(32, .428)=6.688, p<$ 0.0005 . Post hoc tests using the Bonferroni correction revealed that after receiving the group response, participants significantly changed their final response to a more negative one $(-.281): p<.0001$. Looking at the different combinations in the initial group responses, it seems that at least one negative response within the group is needed for participants to change their minds significantly $(t(32)=4.563, p<.0005)$. Hence, no majority of negative group responses is needed for participants to change their final response, but solely one negative response is sufficient.

A second repeated measures ANCOVA was conducted on the initial negative responses (i.e., a no or maybe no response) to adding a song to the playlist. Also in this case the Greenhouse-Geisser correction determined that mean responses on a song differed statistically significantly between time points (i.e., before and after presenting the group responses): $F(1, .692)=68.689, p<0.0005$. Taking into account the group responses, results showed a significant interaction effect as well: $F(32, .692)=18.521, p<$ 0.0005 . Post hoc tests using the Bonferroni correction showed that participants significantly changed their final response to positive (1.012): $p<.0005$. However, when looking at the different combinations of the initial group responses, the results show that participants only changed their final response when there was a majority of votes (i.e., more than half of the group responses were positive): $t(32)=$ $-2.149, p<.001$

\section{Discussion and Conclusion}

Findings and Discussion

The study results indicate different conformity behavior dependent on a participant's initial liking of a song. First, if a participant originally favored a song, only one negative answer (i.e., not wanting to add the song to the group playlist) from another group member was needed to increase the probability that a participant would change their final decision (from favoring to not favoring the song). Second, in contrast, if a participant originally voted against a song being added to the playlist, a majority of positive answers from the other group members (i.e., at least three of the four other group members wanted to add the song) was needed to make the participant change their final decision (from not favoring to favoring the song).

The reasons for such behavior have yet to be investigated. One potential explanation is that the preference in favor of a particular song is not overly strong, so that changing one's mind comes easy. However, in the study design, only an unanimous decision in favor of a song would lead to adding it to the playlist; thus, a participant could keep the positive answer and the song would not be included in the playlist because of someone else voting against it. Hence, we speculate that an individual hesitates to reveal to the group to favor a song that the rest of the group does not like. Another potential reason in the specific experiment setting is that there are lots of song alternatives that could be added to a playlist; in other words, if a favored song does not make it to the playlist, this does not involve a high loss because there are many equally valuable alternatives available.

The need of a majority in favor of a song to flip the judgement of a participant who dislikes the song could be accounted for strong feelings against a particular song. In contrast to the low loss of a favored song not being added 


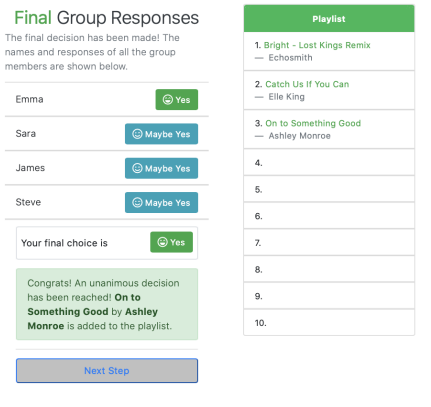

Figure 4: Screenshot showing a song added to a group's playlist.

\section{Acknowledgements}

This research is supported by the Austrian Science Fund (FWF): V579. because of the available alternatives, adding a disliked song to the playlist involves accepting a high loss. Yet, it is interesting to observe that a majority in favor of a song seems to induce participants to take this loss.

Implications

As the switching direction (in favor or against an item) or the involved loss amount seem to play an important role in conformity behavior, our work has theoretical implications for conformity research. Research following Asch's paradigm investigates whether people conform to a majority group opinion against a clear and objectively correct answer. Our experiment using music playlists targets a domain of taste and individual preferences (similar to other domains in entertainment or the fashion domain). Our results suggest for music, being in favor of or against an item leads to different conformity behavior. Our online experiment also differentiates from the study of conformity in discussions of social issues or in the political discourse. Typically, research in those domains investigate conformity in terms of switching between two mindsets in general (e.g., from a conservative to a liberal mindset, or other way round) (e.g., [23, 19]). Potentially the switching direction (e.g., from conservative to liberal), or the loss amount for accepting or discarding a particular single issue associated within the one or the other mindset, may play a similar role in those domains. To the best of our knowledge there is no work that studies conformity on a more fine-grained level; where not only switching (e.g., conservative to liberal in general) is considered, but voiced opinion changes on single issues (e.g., a specific planned measure to counteract the climate crisis) are investigated separately. The expected loss involved in advocating or not to such a specific measure may lead to different conformity behavior. Accordingly, differentiated strategies may be needed to address the different opinions and needs.
Our findings have also implications for recommender systems. Typically, group recommender systems take the group members' preferences as given. Only few studies consider that group members may conform with a majority or an opinion leader. Our findings imply that conformity has to be addressed at a more fine-grained level, considering the switching direction or the loss amount. Our findings also give new direction for sequential recommendations for individuals. We hypothesize that individuals are more willing to accept that a preferred item is not included than accepting a disliked item. Accordingly, a sequence of recommendations (e.g., a playlist) where all included items are perceived as rather okay would be preferred over a set that may include the most favorite item but also disliked ones. This perspective would require the development of novel measures capturing satisfaction with a sequence of recommendations.

\section{Future Work}

Motivated by these insights, we will continue with an indepth investigation on further factors potentially influencing conformity. For example, we asked for familiarity (artist and song) for the suggested songs, as well as satisfaction questions at the end of the study. These factors may provide additional insights on the prerequisites of conformity effects. Additionally, we will investigate cultural differences and further demographics such as gender and age, as these factors have been found influential in earlier research.

Having found that the switching direction leads to different conformity behavior in group playlist creation, we deem worthwhile to investigate whether the direction of opinion or preference change plays a role in other fields, including topics such as the spread of fake news, political debate, and nudging effectiveness. The severity of the expected consequences implied by an opinion change may play a role in future theoretical and empirical pursuits around conformity. 


\section{REFERENCES}

[1] Solomon E Asch. 1955. Opinions and social pressure. Scientific American 193, 5 (1955), 31-35.

[2] Solomon E Asch. 1956. Studies of independence and conformity: I. A minority of one against a unanimous majority. Psychological monographs: General and applied 70, 9 (1956), 1-17. DOI :

http://dx.doi.org/10.1037/h0093718

[3] Solomon E Asch. 1958. Effects of group pressure upon the modification and distortion of judgments. In Readings in social psychology (3rd ed.). Holt, Rinehart and Winston, New York, NY, USA, 174-183.

[4] Rod Bond. 2005. Group Size and Conformity. Group Processes \& Intergroup Relations 8, 4 (2005), 331-354. DOI :

http://dx.doi.org/10.1177/1368430205056464

[5] Rod Bond and Peter B Smith. 1996. Culture and Conformity: A Meta-Analysis of Studies Using Asch's (1952b, 1956) Line Judgment Task. Psychological Bulletin 119, 1 (1996), 111-137. DOI :

http://dx.doi.org/10.1037/0033-2909.119.1.111

[6] Marco Cinnirella and Ben Green. 2007. Does 'cyber-conformity' vary cross-culturally? Exploring the effect of culture and communication medium on social conformity. Computers in Human Behavior 23, 4 (2007), 2011-2025.

[7] Dan Cosley, Shyong K. Lam, Istvan Albert, Joseph A. Konstan, and John Riedl. 2003. Is Seeing Believing? How Recommender System Interfaces Affect Users' Opinions. In Proceedings of the SIGCHI Conference on Human Factors in Computing Systems (CHI '03). Association for Computing Machinery, New York, NY, USA, 585-592. DOI :

http://dx.doi.org/10.1145/642611.642713

[8] Richard S Crutchfield. 1955. Conformity and character. American Psychologist 10, 5 (1955), 191-198. DOI : http://dx.doi.org/10.1037/h0040237

[9] Morton Deutsch and Harold B Gerard. 1955. A study of normative and informational social influences upon individual judgment. The Journal of Abnormal and Social Psychology 51, 3 (1955), 629-636. DOI : http://dx.doi.org/10.1037/h0046408

[10] Sanjeev Dewan, Yi-Jen Ho, and Jui Ramaprasad. 2017. Popularity or proximity: Characterizing the nature of social influence in an online music community. Information Systems Research 28, 1 (2017), 117-136.

[11] Pedro Dias and João Magalhães. 2013. Music Recommendations for Groups of Users. In Proceedings of the 2013 ACM International Workshop on Immersive Media Experiences (ImmersiveMe '13). Association for Computing Machinery, New York, NY, USA, 21-24. DOI :

http://dx.doi.org/10.1145/2512142.2512151

[12] Hauke Egermann, Reinhard Kopiez, and Eckart Altenmüller. 2013. The influence of social normative and informational feedback on musically induced emotions in an online music listening setting.

Psychomusicology: Music, Mind, and Brain 23, 1 (2013), 21-32. DOI :

http://dx.doi.org/10.1037/a0032316 
[13] Alexander Felfernig and Martijn Willemsen. 2018. Handling Preferences. In Group Recommender Systems: An Introduction, Alexander Felfernig, Ludovico Boratto, Martin Stettinger, and Marko Tkalčič (Eds.). Springer International Publishing, Cham, Germany, 91-103. DOI :

http://dx.doi.org/10.1007/978-3-319-75067-5_5

[14] Charles E. Furman and Robert A. Duke. 1988. Effect of Majority Consensus on Preferences for Recorded Orchestral and Popular Music. Journal of Research in Music Education 36, 4 (1988), 220-231. DOI : http://dx.doi.org/10.2307/3344875

[15] Nicholas Hertz, Tyler Shaw, Ewart J. de Visser, and Eva Wiese. 2019. Mixing It Up: How Mixed Groups of Humans and Machines Modulate Conformity. Journal of Cognitive Engineering and Decision Making 13, 4 (2019), 242-257. DOI :

http://dx.doi.org/10.1177/1555343419869465

[16] Yili Hong, Ni Huang, Gord Burtch, and Chunxiao Li. 2016. Culture, conformity, and emotional suppression in online reviews. Journal of the Association of Information Systems 17, 11, Article 2 (2016), 21 pages. DOI :

http://dx.doi.org/10.17705/1jais. 00443

[17] Matthew J Hornsey, Louise Majkut, Deborah J Terry, and Blake M McKimmie. 2003. On being loud and proud: Non-conformity and counter-conformity to group norms. British journal of social psychology 42, 3 (2003), 319-335

[18] Howard G. Inglefield. 1972. Conformity behavior reflected in the musical preference of adolescents. Contributions to Music Education 1 (Autumn 1972), 56-67. DOI: http://dx.doi.org/10.2307/24127367
[19] Meagan Kelly, Lawrence Ngo, Vladimir Chituc, Scott Huettel, and Walter Sinnott-Armstrong. 2017. Moral conformity in online interactions: rational justifications increase influence of peer opinions on moral judgments. Social Influence 12, 2-3 (2017), 57-68. DOI :

http://dx.doi.org/10.1080/15534510.2017.1323007

[20] Eun-Ju Lee. 2006. When and how does depersonalization increase conformity to group norms in computer-mediated communication?

Communication Research 33, 6 (2006), 423-447. DOI : http://dx. doi . org/10.1177/0093650206293248

[21] Min Kyung Lee and Su Baykal. 2017. Algorithmic Mediation in Group Decisions: Fairness Perceptions of Algorithmically Mediated vs. Discussion-Based Social Division. In Proceedings of the 2017 ACM Conference on Computer Supported Cooperative Work and Social Computing (CSCW '17). Association for Computing Machinery, New York, NY, USA, 1035-1048. DOI : http://dx.doi. org/10.1145/2998181.2998230

[22] Yiming Liu, Xuezhi Cao, and Yong Yu. 2016. Are You Influenced by Others When Rating?: Improve Rating Prediction by Conformity Modeling. In Proceedings of the 10th ACM Conference on Recommender Systems (RecSys '16). Association for Computing Machinery, New York, NY, USA, 269-272. DOI : http://dx.doi.org/10.1145/2959100.2959141

[23] Daniel J. Mallinson and Peter K. Hatemi. 2018. The effects of information and social conformity on opinion change. PLOS ONE 13, 5, Article e0196600 (05 2018), 22 pages. DOI :

http://dx.doi.org/10.1371/journal.pone. 0196600 
[24] Misa Maruyama, Scott P. Robertson, Sara Douglas, Roxanne Raine, and Bryan Semaan. 2017. Social Watching a Civic Broadcast: Understanding the Effects of Positive Feedback and Other Users' Opinions. In Proceedings of the 2017 ACM Conference on Computer Supported Cooperative Work and Social Computing (CSCW '17). Association for Computing Machinery, New York, NY, USA, 794-807. DOI : http://dx.doi.org/10.1145/2998181.2998340

[25] Misa T. Maruyama, Scott P. Robertson, Sara K. Douglas, Bryan C. Semaan, and Heather A. Faucett. 2014. Hybrid Media Consumption: How Tweeting during a Televised Political Debate Influences the Vote Decision. In Proceedings of the 17th ACM Conference on Computer Supported Cooperative Work \& Social Computing (CSCW '14). Association for Computing Machinery, New York, NY, USA, 1422-1432. DOI : http://dx.doi.org/10.1145/2531602.2531719

[26] Judith Masthoff. 2015. Group Recommender Systems: Aggregation, Satisfaction and Group Attributes. In Recommender Systems Handbook (2nd ed.), Francesco Ricci, Lior Rokach, and Bracha Shapira (Eds.). Springer US, Boston, MA, USA, 743-776. DOI : http://dx.doi.org/10.1007/978-1-4899-7637-6_22

[27] Paul R Nail, Geoff MacDonald, and David A Levy. 2000. Proposal of a four-dimensional model of social response. Psychological Bulletin 126, 3 (2000), 454.

[28] JungKun Park and Richard Feinberg. 2010. E-formity: consumer conformity behaviour in virtual communities. Journal of Research in Interactive Marketing 4, 3 (2019/12/23 2010), 197-213. DOI : http://dx.doi.org/10.1108/17505931011070578
[29] Tom Postmes, Russell Spears, and Martin Lea. 2000. The formation of group norms in computer-mediated communication. Human communication research 26, 3 (2000), 341-371.

[30] Tom Postmes, Russell Spears, Khaled Sakhel, and Daphne De Groot. 2001. Social influence in computer-mediated communication: The effects of anonymity on group behavior. Personality and Social Psychology Bulletin 27, 10 (2001), 1243-1254.

[31] Lara Quijano-Sanchez, Juan A. Recio-Garcia, Belen Diaz-Agudo, and Guillermo Jimenez-Diaz. 2013. Social Factors in Group Recommender Systems. ACM Transactions on Intelligent Systems and Technology 4, 1, Article 8 (Feb. 2013), 30 pages. DOI : http://dx.doi.org/10.1145/2414425.2414433

[32] Michael Rosander and Oskar Eriksson. 2012. Conformity on the Internet - The role of task difficulty and gender differences. Computers in Human Behavior 28, 5 (2012), 1587-1595. https://doi.org/10.1016/j.chb.2012.03.023

[33] Michael Smilowitz, D Chad Compton, and Lyle Flint. 1988. The effects of computer mediated communication on an individual's judgment: A study based on the methods of Asch's social influence experiment. Computers in Human Behavior 4, 4 (1988), 311-321.

[34] Martin Stettinger, Alexander Felfernig, Gerhard Leitner, and Stefan Reiterer. 2015. Counteracting Anchoring Effects in Group Decision Making. In User Modeling, Adaptation and Personalization (UMAP 2015), Francesco Ricci, Kalina Bontcheva, Owen Conlan, and Séamus Lawless (Eds.). Springer International Publishing, Cham, Germany, 118-130. 
[35] Michail Tsikerdekis. 2013. The effects of perceived anonymity and anonymity states on conformity and groupthink in online communities: A Wikipedia study. Journal of the American Society for Information Science and Technology 64, 5 (2013), 1001-1015. DOI : http://dx.doi.org/10.1002/asi. 22795

[36] Edwin J.C. van Leeuwen, Rachel L. Kendal, Claudio Tennie, and Daniel B.M. Haun. 2015. Conformity and its look-a-likes. Animal Behaviour 110 (2015), e1-e4. DOI :

http://dx.doi.org/10.1016/j.anbehav. 2015.07.030

[37] Lisa Slattery Walker. 2015. Social Influence. In The Blackwell Encyclopedia of Sociology. American Cancer Society. DOI : http://dx.doi.org/10.1002/ 9781405165518.wbeoss154.pub2

[38] Senuri Wijenayake, Niels van Berkel, Vassilis Kostakos, and Jorge Goncalves. 2019. Measuring the Effects of Gender on Online Social Conformity. Proceedings of the ACM Human-Computer Interaction 3, CSCW, Article 145 (Nov. 2019), 24 pages. DOI : http://dx.doi.org/10.1145/3359247
[39] Stephan Winter, Caroline Brückner, and Nicole C. Krämer. 2015. They Came, They Liked, They Commented: Social Influence on Facebook News Channels. Cyberpsychology, Behavior, and Social Networking 18, 8 (2015), 431-436. DOI : http://dx.doi.org/10.1089/cyber.2015.0005

[40] Kexin Zhao, Antonis C. Stylianou, and Yiming Zheng. 2018. Sources and impacts of social influence from online anonymous user reviews. Information \& Management 55, 1 (2018), 16-30. DOI : http://dx.doi.org/10.1016/j.im.2017.03.006

[41] Haiyi Zhu, Bernardo Huberman, and Yarun Luon. 2012. To Switch or Not to Switch: Understanding Social Influence in Online Choices. In Proceedings of the SIGCHI Conference on Human Factors in Computing Systems (CHI '12). Association for Computing Machinery, New York, NY, USA, 2257-2266. DOI :

http://dx.doi.org/10.1145/2207676.2208383 